SECTION 4. Computer science, computer engineering and automation.

Tolstova Irina Vladimirovna

a master's student of Department «Radioelectronic systems» Research Institute «Digital signal processing and computer vision»

Don state technical University, Russia Semenishchev Evgeniy Aleksandrovich candidate of technical Sciences,

associate Professor of Department «Radioelectronic systems» Research Institute «Digital signal processing and computer vision»

Don state technical University, Russia

Katkov Dmitriy Nikolayevich student of Department «Radioelectronic systems» Research Institute «Digital signal processing and computer vision» Don state technical University, Russia

\title{
DEVELOPMENT AND INVESTIGATION OF THE MERGE ALGORITHM IMAGE WITH AUTOMATIC CORRECTION OF COLOR
}

The purpose of work is development and research merge algorithm images with automatic color correction.

Keywords: color, automation, algorithms.

\section{РАЗРАБОТКА И ИССЛЕДОВАНИЕ АЛГОРИТМА ОБЪЕДИНЕНИЯ ИЗОБРАЖЕНИЙ С АВТОМАТИЧЕСКОЙ КОРРЕКЦИЕЙ ЦВЕТА}

Целью работы является разработка и исследование алгоритма объединения изображений с автоматической коррекцией ивета.

Ключевые слова: корекция цุвета, автоматизация, алгоритмы.

Современные автоматизированные системы и комплексы, построенные на основе объединѐнных фото и видео потоков, получаемых с выходов светочувствительных матриц, требуют комплексного подхода к обработке и анализу получаемых данных. Особое значение для анализа визуальной информации имеют объединенные изображения мозаики, позволяющие наблюдать непрерывную сцену целиком вместо просмотра разрозненных ее частей. Задача получения объединѐнных изображений является актуальной, поскольку необходимость совмещения возникает: в охранных системах и комплексах предназначенных для наблюдения за территорией; при объединении медицинских снимков, в рентгенограммах, 
клеточных структурах для выявления патологий и развитии течения биологических процессов; при построении картографических изображений, полученных со спутников или летательных аппаратов; при решении задач фотограмметрии; при получении обзорных снимков, используемых в строительстве и т.д. [1, с. 984-986]

Большинство используемых на практике алгоритмов не являются универсальными и в ряде случаев требуют участие оператора при выборе параметров и перенастройке системы. Существующие методы и алгоритмы объединения используемые, например, для панорамного фотографирования или получения общего изображений местности со спутника используют фиксированные значения выдержки и угла фокуса. Однако в случае невозможности получения кадров из одной точки или получение снимков в «ближней» зоне с изменением выдержки делает большинство алгоритмов неспособных $к$ совмещению в единую композицию без участия оператора. Получение изображений разного масштаба, также накладывает ограничения на использование используемых алгоритмов, а в ряде случаев делает невозможным их применение. В связи, с чем разработка новых методов и алгоритмов объединения изображений в единую композицию является актуальной задачей.

Целью работы является разработка и исследование алгоритма объединения изображений с автоматической коррекцией цвета.

Применяемые алгоритмы для объединении фотографий в панорамный вид не учитывают разницу в масштабах, возможность объединения изображений, полученных с различных точек фиксации, изменение интенсивности освещѐнности, коррекцию контраста и цвета. Для дополнения представленных возможностей в данной работе предложен алгоритм объединения изображений в мозаику (рис.1).

Предложенный алгоритм объединения изображений в мозаику реализуется следующим образом. На первом этапе производится загрузка изображений. На втором этапе алгоритма осуществляется выделение границ. Данный шаг служит для реализации задачи поиска детализированных объектов на изображении. Применяется детектор Канни (John Canny), который показывает наилучшие визуальные и количественные результаты при работе, как на чистых изображениях, так и изображениях, подверженных различным искажениям [2, с. 374-377.].

При поиске детализированных объектов методом «Плотность» проводится аналогия с физическим пониманием термина «плотность» в некотором объеме. То есть вначале считается общий коэффициент детализации на всем изображении, который определяется по формуле (1):

$$
\frac{\sum I(x, y)}{i \cdot j}=P_{\text {обиий }}
$$


где $I(x, y)$ - значение пикселя с координатами х и у; $\mathrm{i}$ - количество строк; $\mathrm{j}$ - количество столбцов.

Данный коэффициент детализации определяется автоматически для каждого изображения и его подбор не требует эмпирического вмешательства.

Далее вычисляется плотность в каждом скользящем окне, как показано в выражении (2):

$$
\frac{\sum I(x, y)}{0.1 \cdot i \cdot j}=P_{\text {окна }}
$$

где 0,1 - коэффициент усреднения, связанный с автоматическим выбором размера окна, равного $10 \%$ от общего изображения.

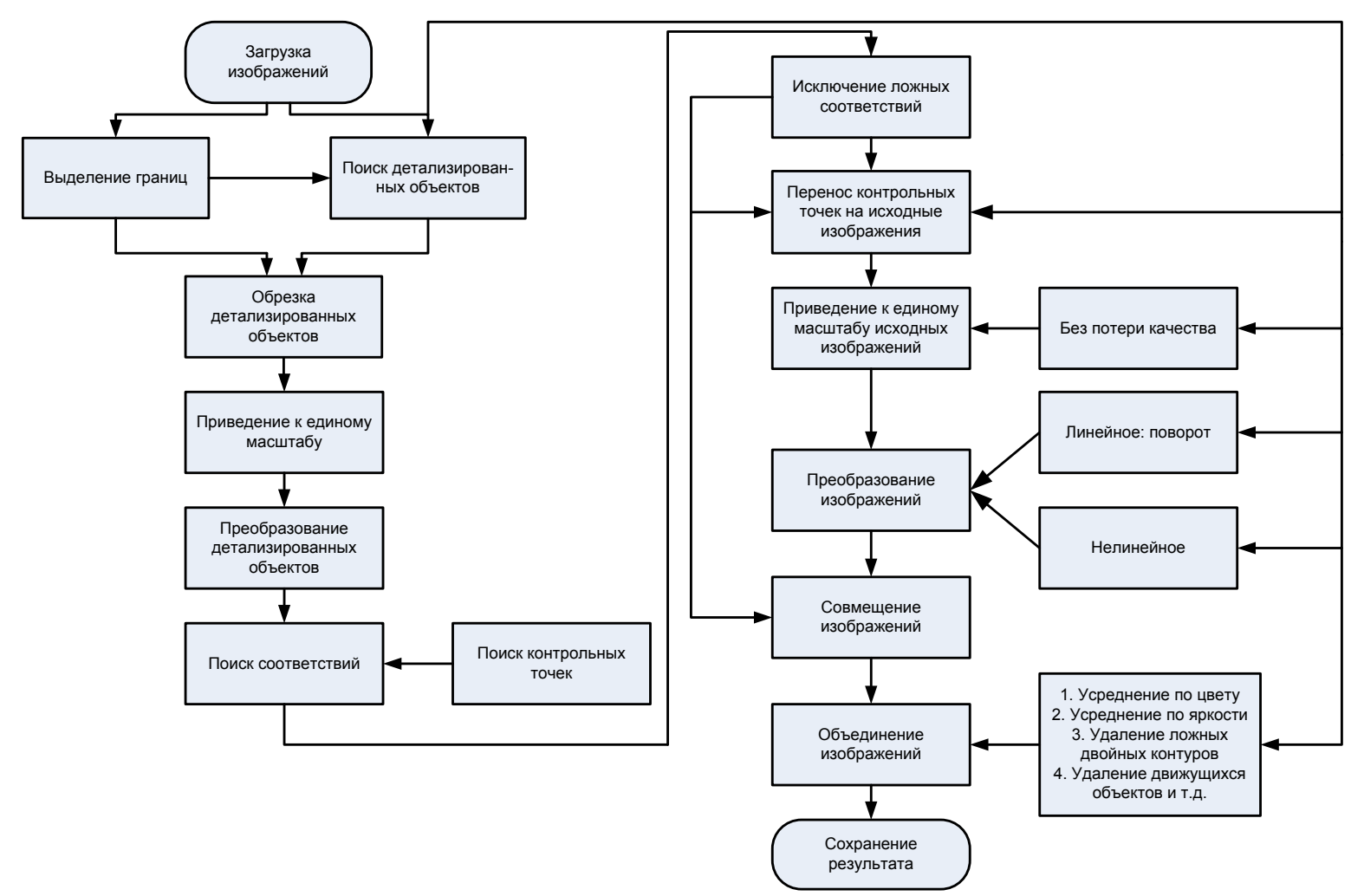

\section{Рисунок 1- Алгоритм объединения изображений в мозаику.}

Далее $P_{\text {общий }}$ и $P_{\text {окна }}$ сравниваются, принимается решение о детализированности в данном окне, и окно сдвигается влево[3, с. 796-800].

Следующим шагом реализации алгоритма является локализация областей с детализированными объектами, в связи с тем, что ряд операций будет производиться именно с определѐнными участками.

В полученных, на прошлом этапе, высокодетализированных областях определяется гистограммы яркости по каждому из каналов. Пример определения гистограмм приведѐн на рисунке 2. 
Для реализации поиска контрольных точек и их соответствий, применяется метод SURF (Speeded Up Robust Features) [4, c. 346-359,]. Данный подход ищет особые точки на изображении и создает их дескрипторы, инвариантные к масштабу и вращению. Это значит, что описание ключевой точки будет одинаково, даже если образец изменит размер, и будет повернут.

Следующим шагом является исключение ложных соответствий. В процессе поиска соответствий полученных контрольных точек разными методами, кроме истинных точек определяются и ложные точки. Для исключения ложных соответствий в работе используется метод RANSAC [5. c. p. 381-395].

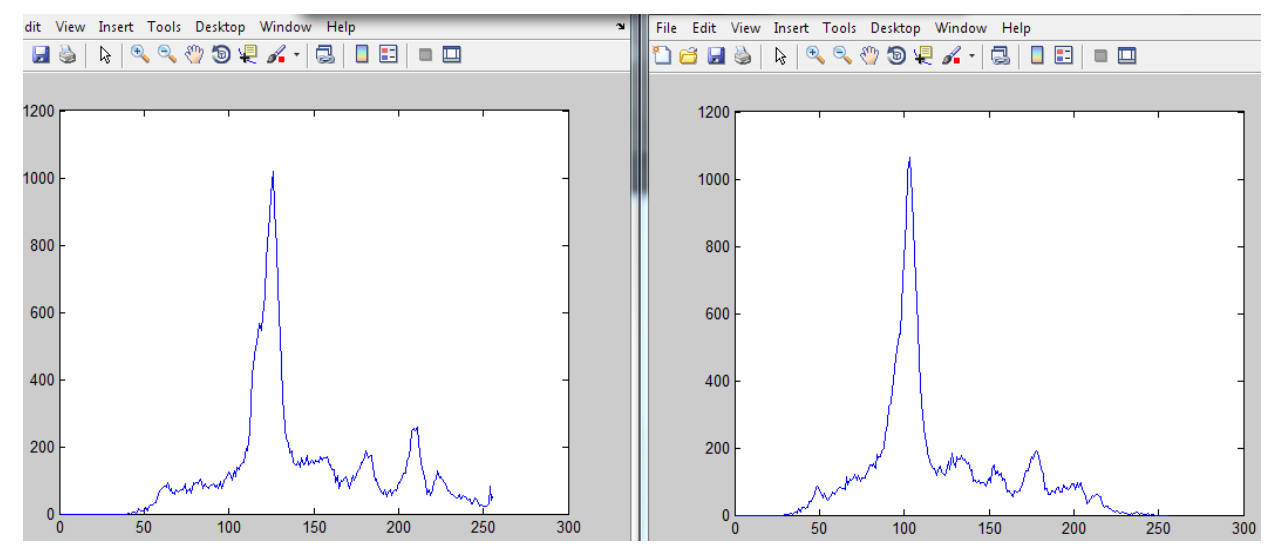

\section{Рисунок 2 - Пример определения гистограмм яркости для высокодетализированных областей.}

Получив соответствия, определенные контрольные точки переносятся на исходные изображения. По данным контрольным точкам и будет происходить непосредственно объединение.

На следующем шаге реализуется приведение детализированных объектов к единому масштабу и нахождение коэффициента масштабирования. Данный коэффициент показывает, во сколько раз объекты различаются по размеру между собой. Данный коэффициент, даѐт возможность применить его ко всему изображениям без изменения размеров высокодетализированных участков.

Для выполнения операции выравнивая изображений по гистограммам, по определѐнным и сопоставленным высокодетализированным областям строятся гистограммы яркости (рис. 2). Полученные гистограммы пропорционально сравниваются с гистограммами всего изображения. Путѐм домножения одного из объединяемых изображений на полученный коэффициент определѐнный в каждом из цветовых каналов происходит выравнивание цветовой палитры в последующем объединяемом изображении. 
Этап «преобразование детализированных объектов» позволяет произвести локальную геометрическую коррекцию между полученными детализированными объектами и получить коэффициент преобразования. Коэффициент преобразования между детализированными объектами, можно применить в дальнейшем к одному или более объединяемым изображениям.

Выбор преобразования является следующим шагом алгоритма. Есть вероятность, что для одного изображения будет недостаточно одного преобразования, вследствие чего преобразования будут комбинироваться. Соответственно, для одного изображения будет несколько коэффициентов преобразования. Данные коэффициенты необходимо «усреднить» и получить общий коэффициент для всего изображения. В большинстве случаев достаточно двух преобразований.

На этапе совмещения изображений необходимо правильное наложение областей объединения. Совмещение производится по найденным контрольным точкам и их соответствиям на обоих изображениях, с исключенными ложных соответствий.

Пример объединения изображений по разработанному в работе алгоритму представлен на рисунке 3.

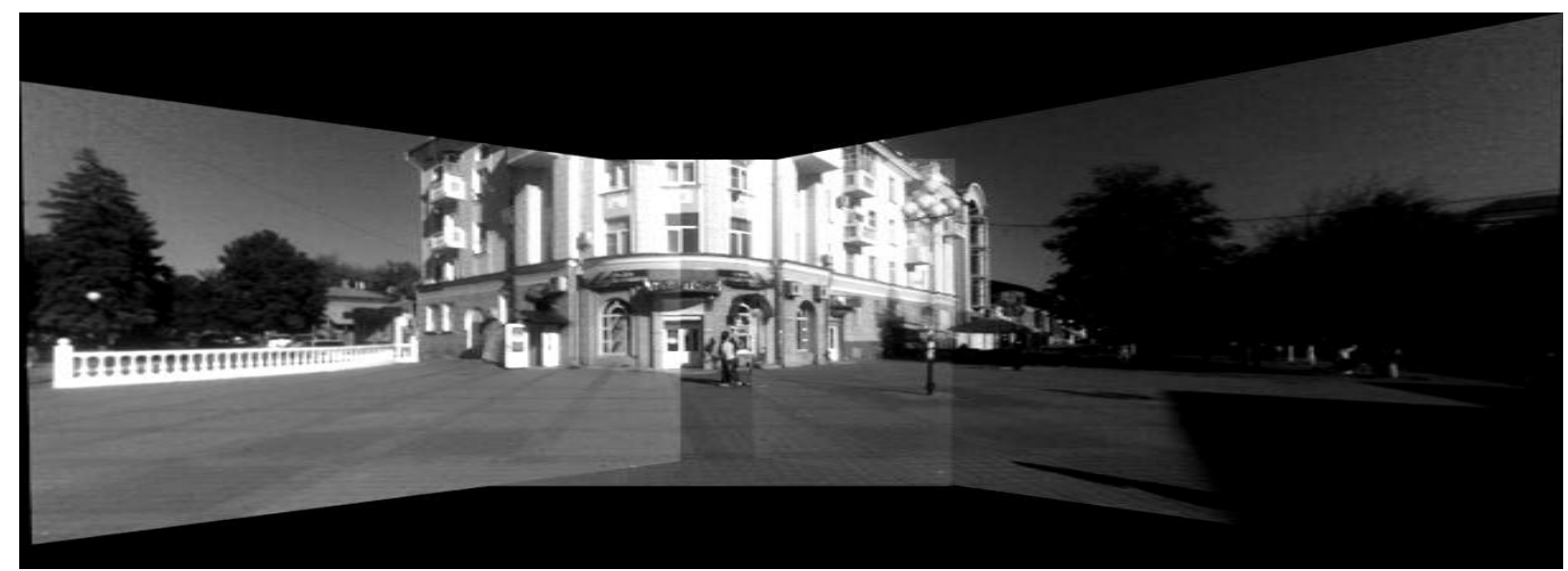

Рисунок 3 - Пример объединения изображний предложенным в работе алгоритмом.

В результате проведѐнных исследований разработан алгоритм объединения изображений в мозаику с возможностью как геометрической коррекции объединяемых объектов, так и яркостной.

\section{Литература}

1. Гонсалес, Р. Цифровая обработка изображений / Р.Гонсалес, Р. Вудс M.: Техносфера, 2005. - 1072 c.

2. Семенищев, Е.А. Разработка и исследование методов выделения 
высокодетализированных объектов на изображениях / Семенищев Е.А., Тазетдинова Д.И., Писарев А.В., Жук С.В., Тарасов Д.А. // Научно-технический вестник Поволжья. 2012. № 6.

3. Семенищев, Е.А. Выделение локальных участков высокодетализированных объектов на общем изображении сцены// Семенищев Е.А., Марчук В.И., Торопов И.А., Толстова И.В/ Нелинейный мир.// №11, т.10, 2012 год.

4. Herbert Bay, Andreas Ess, Tinne Tuytelaars, Luc Van Gool, "SURF: Speeded Up Robust Features", Computer Vision and Image Understanding (CVIU), Vol. 110, No. 3, 2008

5. Martin A. Fischler and Robert C. Bolles, « Random Sample Consensus: A Paradigm for Model Fitting with Applications to Image Analysis and Automated Cartography », Comm. Of the ACM, vol. 24, juin 1981. 